\title{
Experimental Models of Acute Lung Injury
}

\author{
Emine Yllmaz Sipahi ${ }^{1}$, Figen Atalay ${ }^{2}$
}

${ }^{1}$ Department of Medical Pharmacology, Bülent Ecevit University Faculty of Medicine, Zonguldak, Turkey

${ }^{2}$ Department of Pulmonary Medicine, Bülent Ecevit University Faculty of Medicine, Zonguldak, Turkey

\begin{abstract}
Although many various models have been improved in order to form human features of Acute Respiratory Distress Syndrome (ARDS) in animals, there is no single animal model that satisfactorily exhibits all of the histopathological components of human ARDS. Therefore, when choosing an ARDS animal model, it is important to consider the ARDS key property to be tested as a study hypothesis and choose the most appropriate model that can display that property. After mentioning the reasons and physiopathology of ARDS briefly, the purpose of this review is to summarise the most common experimental animal models and the features of these models.
\end{abstract}

Keywords: Acute lung injury, ARDS, experimental models

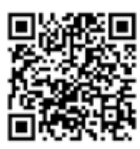

Received date: 02.02 .2013 Accepted date: 20.05 .2013

\section{Address for correspondence} Figen Atalay, Department of Pulmonary Medicine, Bülent Ecevit University Faculty of Medicine, Zonguldak, Turkey

E-mail: figendr@hotmail.com

(C) Copyright 2014 Turkish Respiratory Society (TRS) DOI: 10.5152/ejp.2014.49091

- Available online at www.eurasianjpulmonol.com

\section{INTRODUCTION}

Acute Lung Injury (ALI) is an acute and persistent lung inflammation accompanied by increased vascular permeability. The more severe and advanced form of ALI is defined as Acute Respiratory Distress Syndrome (ARDS). ALI/ARDS was first described by Ashbaugh et al. (1) in 1967.

For acute lung injury/acute respiratory distress syndrome, the definitions that had been made at American-European Consensus Conference on ARDS in 1994 were used until 2012 Berlin definition $(2,3)$. The main alteration of the Berlin definition is the addition of a minimal ventilator setting as well as the removal of the term "acute lung injury" and the pulmonary artery wedge pressure criterion. Accordingly, the following are the primary clinical features used to diagnose ARDS:

1. Having respiratory symptoms in the last week or the symptoms getting worse compared to previous weeks, or developing new symptoms,

2. Having an image consistent with bilateral pulmonary edema on chest radiography or computed tomography of the thorax (CT),

3. Having unexplained respiratory failure by cardiac insufficiency or fluid overload [hydrostatic pulmonary edema must be excluded by echocardiography (ECHO)],

4. Having moderate or severe oxygenation defects $\left(\mathrm{PaO}_{2} / \mathrm{FiO}_{2}\right)$. The severity of hypoxemia determines the severity of ARDS.

a. Mild ARDS: $\mathrm{PaO}_{2} / \mathrm{FiO}_{2}>200 \mathrm{mmHg}$, but $\leq 300 \mathrm{mmHg}$, ventilator setting positive end-expiratory pressure (PEEP) or continuous positive airway pressure (CPAP) $\geq 5 \mathrm{~cm} \mathrm{H}_{2} \mathrm{O}$

b. Moderate ARDS: $\mathrm{PaO}_{2} / \mathrm{FiO}_{2}>100 \mathrm{mmHg}$, but $\leq 200 \mathrm{mmHg}$, ventilator setting PEEP $\geq 5 \mathrm{~cm} \mathrm{H}_{2} \mathrm{O}$

c. Severe ARDS: $\mathrm{PaO}_{2} / \mathrm{FiO}_{2} \leq 100 \mathrm{mmHg}$ ventilator setting $\mathrm{PEEP} \geq 5 \mathrm{~cm} \mathrm{H}_{2} \mathrm{O}$

Despite the new treatment methods provided by the technological advances, acute respiratory distress syndrome has been one of the most mortal diseases in intensive care units with a high mortality rate for the last four decades. Although the mortality rate of ARDS patients is cited to lie within the range of $40 \%$ to $60 \%$, the severity of lung injury or extrapulmonary disease and its concomitant factors also plays an important role in prognosis $(4,5)$. 
To date, more than 60 causes of ARDS have been identified. However, epidemiological studies have shown that in most cases, several prevalent causes may be effective alone or in combination. The most common causes of ARDS are sepsis, pneumonia, aspiration of gastric contents, trauma, and urgent transfusions. Furthermore, overdose medication (aspirin, cocaine, opioids, tricyclic antidepressant drugs, and radiographic contrast agent), cardiopulmonary bypass, pneumonectomy, and acute pancreatitis are among the rarer ARDS causes (6-10).

Acute respiratory distress syndrome is non-cardiogenic pulmonary edema secondary to increased permeability. In ARDS, accumulated alveolar edema is mainly caused by a damaged alveolar (epithelial)-capillary (endothelial) barrier and increased permeability. Acute respiratory distress syndrome is an acute clinical condition that may occur when the lungs are subjected to various factors via airways and circulation. At the American-European Consensus Conference, ARDS was defined as inflammation and increased permeability syndrome and it was suggested that the damage to the alveolar-capillary structure was caused by aggressive inflammatory reactions (2).

In a healthy lung, circulation is organised to ensure that there is very little fluid in the interstitial space and to prevent accumulation of fluid in the alveoli. The oncotic pressure of intravascular proteins and tight junctions that prevent leaking in the alveoli along with interstitial lymphatics preserve this balance. However, as a result of lung injury, these mechanisms collapse and excessive accumulation of fluid in alveoli and interstitium takes place; in the meantime, proteins start leaking out of vessels into the alveoli and interstitium. Once this accumulation exceeds the capacity of the interstitium and lymphatics to drain, the alveoli are filled with bloody, proteinosis edema fluid and dead cells. As a result of surfactant dysfunction, since surface tension cannot be decreased, alveoli collapse. Eventually, pulmonary compliance decreases, breathing becomes laboured and the subsequent deterioration in oxygenation takes place, before respiratory distress presents itself. This pathophysiological process results in severe respiratory distress $(4,10-12)$.

Complicated processes that develop in acute lung injury begin following a triggering event after some cellular and chemical mediators become activated. These mediators cause free oxygen products and toxic enzymes to emerge for tissues that are released from cells, especially from neutrophils. In the end, a sequence of events leading to alveolar and capillary damage begins. Whatever the situation that initiated the event is, it is remarkable that the pathological changes do not differ. Various biological processes such as inflammation, apoptosis and thrombosis take active roles in the pathogenesis of acute respiratory distress syndrome $(5,9,11)$.

For incidents that develop in the pathogenesis of acute respiratory distress syndrome, it is thought that numerous cellular and biochemical mediators play a role. It is known that cytokines, which are activated by stimulated complement-mediated neutrophils, play a major role. As a response to various stimulants, proinflammatory cytokines such as TNF-alpha, interleukin-1, interleukin- 6 and interleukin-8 are secreted. Proinflammatory cytokines can be generated from inflammatory cells in the lungs, fibroblasts and epithelial cells of the lungs. Afterwards, substantial neutrophil accumulation takes place in the lungs and these cells become activated and secrete toxic mediators such as reactive oxygen derviatives and protease, which lead to capillary endothelial and alveolar epithelium damage. Like neutrophils, proteolytic enzymes and reactive oxygen derivatives are secreted from alveolar and interstitial macrophages in the lung tissue, which causes tissue damage. Activated arachidonic acid metabolites (platelet activating factor), tromboxane- $\mathrm{A}_{2}\left(\mathrm{TXA}_{2}\right)$, and leukotriene- $\mathrm{B}_{4}\left(\mathrm{LTB}_{4}\right)$ cause increased permeability and cellular damage. Nitric oxide (NO), which is generated in many cells, especially in sepsis, is a strong endogenous vasodilator; with increased permeability and reactive oxygen species, it plays an active role in alveolar and capillary damage $(9,11,13-18)$. None of the processes that have been identified to date are entirely responsible for the development of ARDS alone and it involves complicated relationships that cannot still be exactly explained.

\section{ALI/ARDS Experimental Models}

The studies that have been performed for ARDS in humans give vital information about the onset and development of physiological and inflammatory changes in the lungs. This information enables hypotheses on injury mechanisms to be developed; however, for critically-ill patients, the presence of a wide range of clinical variables, which is difficult to control, has proven that testing these hypotheses on humans is extremely challenging. Animal models lay a bridge between patients and laboratory findings. On the other hand, the criteria of the "American-European Consensus Conference on ARDS" cannot be applied directly to animal experiments. Procedures such as arterial blood gas, chest radiography, cardiac echography and even catheterisation can be implemented on small experimental animals, but procedures can be performed only in a few laboratories. In addition, these measurements may be impossible to make for many experimental methods. Another approach to identify ALI in animals is using the histopathological criteria observed ALI in humans. However, there is no experimental animal model that completely imitates inflammatory infiltration, alveolar wall thickening, and hyaline membrane formation with characterised diffuse alveolar damage determined in human ALI.

There are 3 key pathological features of human ALI and ARDS. The components of this pathological trio are a neutrophilic alveolitis, hyaline membrane accumulation and microthrombus formation. An ideal "ALI/ARDS animal model" is a model that is formed by this pathological trio. Nevertheless, there is no ideal model that completely imitates human pathology and involves all of these features (19). Lacking one of these factors does not mean that this model is not a form of acute lung injury. In animal models, which pathologies make that model an "experimental acute lung injury" or determining whether a medication that fixes one of the damages in pathogenesis but is ineffective to the others (e.g. effective for decreasing neutrophil migration to lungs but ineffective in permeability) can prevent lung injury or not is debatable. Besides, it is challenging to make comparisons because of the diversity of tests used by researchers (20). Thus, in experimental studies, it would be a better approach to choose the model in which the effects of the factors that you want to study are activated.

\section{What should be done with animal models?}

1. The animal models should imitate one or more physiological and pathological mechanisms and the outcomes of human ALI (detection of rapid development process measured in hours, gas 
exchange abnormalities, decreased pulmonary compliance, increased permeability in alveolar- capillary membrane)

2. Since the response given by the lung varies, the same amount of damage and repair phase should be provided (before being diagnosed with clinical ALI in humans, intrapulmonary inflammatory response is received and the first three days of ALI/ARDS are severe). Animal experiments should be prolonged if the animal can be supported.

3. The human lung is affected by therapies (e.g. mechanical ventilation) as well as primary disease (e.g. sepsis). These applications should be added to animal experiments if possible.

The factors pertaining to animal experimental models mentioned here cannot be fulfilled completely, so there is no experimental animal model that exactly imitates ALI/ARDS observed in humans. In this case, the question is how many of these factors are necessary for the animal in order to diagnose ALI. This is a complicated situation, with no right or wrong answer and it depends on many factors including the criteria specifically for the experimental model (20). One of the underlying reasons for not being able to imitate human pathology is the biological differences between humans and animals. Various animal species are used in order to study lung injury mechanisms, but in terms of response given to the injury, especially given to microbial structure, some vital and sometimes major differences are detected among animal species. Here are some examples related to species differences for immune response:

1. Toll-like receptor (TLR) differences; TLR4, responsible for the detection of microbial structures, can detect different lipopolysaccharide (LPS) structures in humans and mice. Similarly, for TLR2, $3,7,8$ and 9 , species differences are present (21).

2. Species differences in mononuclear phagocytic system (MPS); this system consists of macrophages and monocytes. Intravascular macrophages directly encounter microorganisms, endotoxins and circulating particles and destroy them. Circulating macrophages show differences between species. In most species, intravascular macrophages are restricted to organs like the spleen and liver (splenic macrophage, kupffer cell). However, some species' lungs have mature pulmonary intravascular macrophages (PIM), which are non-migratory cells adherent to the endothelium in pulmonary capillary. Bovines, swine, sheep, cats, goats, horses, and marine mammals have PIM (22). While for species with PIM, particles and LPS are localised in the lungs, in species without PIM, the particles are localised in the spleen and liver (dogs, rats, mice, rabbits, non-human primates). Studies have shown that PIM is not a part of MPS in normal individuals (23). When PIM depletion is performed in bovines, it is observed that endotoxin-mediated lung injury decreases (24). Thus, it can be concluded that the presence of PIM increases susceptibility to lung injury. These results should be taken into consideration when creating an animal model for human lung injury.

3. Nitric oxide (NO) differences among species; NO takes an active role in lung injury pathogenesis. For rodents and humans, the ability to produce NO varies significantly (19). While rodent macrophage produces a great deal of NO, for humans, unactivated macrophages produce very little NO. Bovine macrophages produces NO, but just like humans, hamster, monkey, goat and swine macrophages produce very little NO (25).

4. Differences in chemokines and chemokine receptors; CXCL8/IL-8 is a powerful neutrophil chemotactic factor and takes an active role in ALI pathogenesis. CXCL8 and the related CXC-chemokines are produced in all species against bacterial products. There is no CXCL8 gene in rats and mice, but two related CXC-chemokines are produced [KC and macrophage inflammatory protein-2 (MIP-2)]. They have more equivalent arrays than CXCL1-3/GRO $\alpha$, $\beta$ and $\gamma$ with CXCL8 in humans. It is thought that they are functionally homologous with CXCL8 since they play critical roles in lung neutrophil involvement $(19,26)$.

The differences like these between humans and other species may negatively affect the clinical correlation of the experiments that we perform. As a result, as noted below, experimental models' simulation of human pathology and the follow-up process become difficult $(19,20,27)$.

1. Experimental models generally have milder pathology compared to human pathology.

2. The monitored period is shorter the formation of pathology takes hours or days in humans. In this case, the difficulties concerning monitoring are involved. The species and the size of the animal may cause differences in application.

3. Therapeutic agents in experimental studies are given before the onset of ALI/ARDS, whereas the clinical diagnosis and treatment of ALI/ARDS is delayed.

4. Animal experiments are performed on young animals; however, human patients are mostly elderly and may have many medical problems such as diabetes, coronary artery disease, kidney or liver failure.

5. Biochemical markers (parameters, tests) measured in bronchoalveloar lavage fluid (BAL), plasma and edema fluid may not correlate with their biological activities (markers may not correlate with clinical diagnosis and pathogenesis, while these factors take part in extracellular transmission, they may not be released extracellularly and may not be measured).

Numerous different model strategies have been improved in animals to create human ALI features. It is possible to divide these models into two groups as direct or indirect lung injury models (Table 1) (28).

\section{The models of direct lung injuries caused by injurious stimulus: This} group involves intratracheally or intranasally bacteria or a bacterial yield such as LPS; acid yield such as hydrochloric acid $(\mathrm{HCl})$ or gastric particle to create an acid aspiration; oxygen yield in high inspired fraction; surfactant depletion via rapid lavages with $0.9 \%$ sodium chloride $(\mathrm{NaCl})$; forming ischaemia/reperfusion by clamping hilus or exposure to mechanical stress via mechanical ventilation at high tidal volumes $(19,20,28)$.

Indirect lung injuries: this category generally includes models based on the formation of sepsis. Some of them are caecal ligation and puncture, intravenously bacteria or LPS yield and mesenteric ischaemia/reperfusion models. This category also includes oleic acid depending on formation of oleic acid keratosis from bone marrow for patients with multiple bone fractures $(19,20,28)$. Acute lung injury that is created by intraperitoneal application of ANTU (a-naphthyltio urea), a rodenticide medication, is also an appropriate approach for indirect lung injury (29).

Combination models: In order to create a similar human ALI/ARDS model, various injury strategies can be combined. The most common 
Table 1. Lung injury animal models

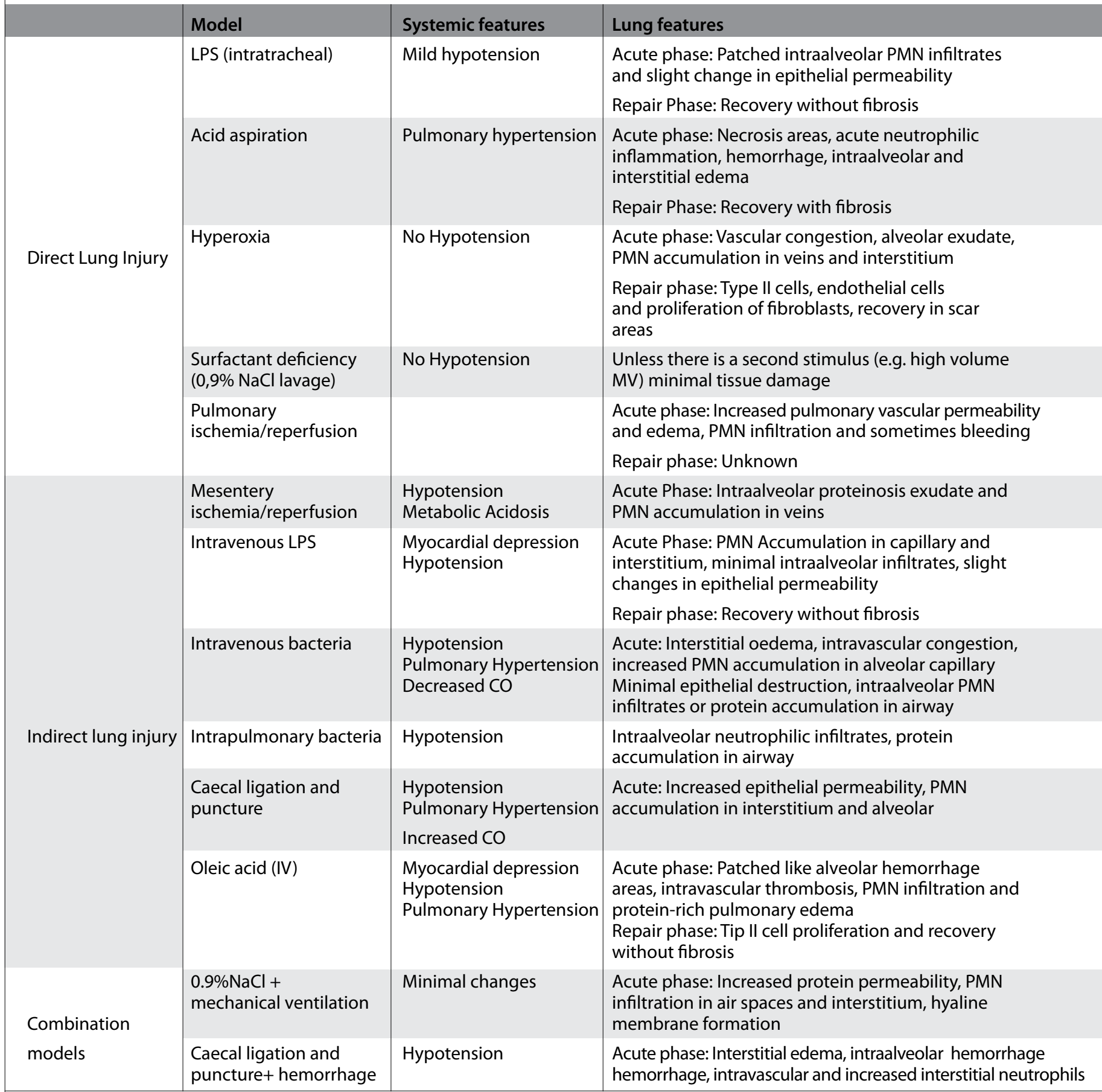

CO: carbon monoxide; IV: intravenous; LPS: lipopolysaccaride; MV: mechanical ventilation; PMN: polymorphonuclear leukocytes

strategies are the performance of mechanical ventilation following $0.9 \% \mathrm{NaCl}$ lavage or the formation of hemorrhage following caecal ligation/puncture operation $(19,20,28)$.

There are 3 vital points in animal models for human respiratory distress syndrome:

1. There is no animal model that completely exhibits all pathological findings of human ALI/ARDS. Although all of the components of ALI/ARDS pathological trio are exhibited, these are generally milder findings compared to human ARDS.
2. The effect of models on each ALI/ARDS pathological trio component is different. For instance, while neutrophilic alveolitis is significant in some models (like the model where LPS is yielded into the lungs), the main feature is an increase in intraalveolar proteinosis material (like the lung ischaemia/reperfusion model) in other models.

3. Sepsis models frequently progress with increased neutrophil accumulation in the pulmonary vasculature and a slight increase in intraalveolar content, but intraalveolar neutrophils with PMN infiltration or protein accumulation is less. In other words, in sep- 
Table 2. Advantages and disadvantages of ALI/ARDS models

\begin{tabular}{|l|l|l|}
\hline $\begin{array}{l}\text { Model } \\
\text { Oleic acid (intravenous) }\end{array}$ & $\begin{array}{l}\text { Advantage } \\
\text { Oleic acid (intravenous) } \\
\text { ALI trio (but in a milder form). Repair } \\
\text { phase can be formed } \\
\text { A good model for sepsis }\end{array}$ & $\begin{array}{l}\text { Disadvantage } \\
\text { Has to be administered intravenously } \\
\text { The relation with the mechanisms is not clear. }\end{array}$ \\
$\begin{array}{l}\text { LPS (intratracheal) } \\
\begin{array}{l}\text { Acid aspiration } \\
\text { Hyperoxia }\end{array}\end{array}$ & $\begin{array}{l}\text { A good model for neutrophil accumulation } \\
\text { A good model for epithelial barrier damage } \\
\text { ALI/ARDS findings (minimal intra-alveoler neutrophil } \\
\text { infiltration and protein-rich alveolar oedema) } \\
\text { Minimal epithelial barrier changes }\end{array}$ \\
$\begin{array}{l}\text { Surfactant deficiency } \\
\text { A good model for hemorrhagic injury }\end{array}$ & $\begin{array}{l}\text { Neutrophil response for human is less significant } \\
\text { Less intraalveolar neutrophil infiltration } \\
\text { compared to human ALI/ARDS } \\
\text { The tissue damage that it creates on its own is very little } \\
\text { reperfusion } \\
\begin{array}{l}\text { Mesenteryischemia/reperfusion } \\
\text { (CLP, intravenous LPS) }\end{array}\end{array}$ & $\begin{array}{l}\text { Tagents; especially with mechanical ventilation } \\
\text { ALI: acute lung injury; ARDS: acute respiratory distress syndrome; CLP: cecal ligation and puncture; LPS: lipopolysaccaride }\end{array}$ \\
\hline
\end{tabular}

sis models, lung injuries are primarily localised in vascular and interstitial compartments and the alveolar space is less affected.

\section{Model Selection}

As mentioned previously, there is no single model that satisfactorily exhibits all of the histopathological components of human ALI/ ARDS. Therefore, when choosing an ALI/ARDS animal model, it is important to consider the ALI/ARDS key feature that is to be studied with a study hypothesis and then select a model that can exhibit those features (Table 2) (28). For example, if the researcher is working on neutrophil influx into the lungs, a model characterised primarily with alveolar neutrophil, such as LPS yield, should be selected. On the contrary, if the researcher is interested in mechanisms of epithelial damage, a model characterised by significant hyaline membrane formation such as lung ischaemia/reperfusion or the acid yield model should be preferred.

Another important point to consider in model selection is selecting animal species. There are some advantages of mouse models. Having numerous genetically modified species allows mechanism studies. However, due to their small size, physiological studies are very difficult, or even impossible. Another problem is that mice are different from humans in many aspects. For instance, mice lack IL-8, but for humans, it is the most important neutrophil chemoattractant. Bigger animals like swine and rabbits have IL-8 and are ideal for complex physiological measurements, but they are rather costly (28).

\section{Experimental Models}

Acid aspiration: Gastric content aspiration is an important risk factor for $\operatorname{ARDS}(30,31)$. The main factor that causes injury in gastric content is low $\mathrm{pH}$. Because of that, hydrochloric acid $(\mathrm{HCl})$ is used to create lung injury in animals. A lot of factors, such as low $\mathrm{pH}$, high osmolarity, food remains in aspired oropharyngeal and gastric content and bacterial products may play an important role in aspiration-induced human acute lung injury pathogenesis (32). Acid damage is created by inject- ing $\mathrm{HCl}$ directly to the trachea or bronchus while the animal is mechanically ventilated. Although hydrochloric acid is rapidly hydrolysed in the lungs, $\mathrm{HCl}$ concentration affects the degree of lung injury (28). For most studies, at a concentration of $0.1 \mathrm{~N}, \mathrm{HCl}$ (up to $0.5 \mathrm{~N}$ ) has been used. Because the $\mathrm{pH}$ value that is used is generally lower than that of the gastric juice of intensive care unit patients (mostly between $\mathrm{pH} 3$ and 4), another approach is to titrate $0.3 \% \mathrm{NaCl}$ with $\mathrm{HCl}$ until the $\mathrm{pH}$ is 1.2-1.5 and to obtain a closer result in terms of both $\mathrm{pH}$ and osmolarity $(19,28)$.

Low pH acid aspiration is a neutrophil-mediated lung injury. It is characterised by damage in the airway and alveolar epithelium; damage takes place in type I alveolar epithelium and then a repair phase progresses with type II proliferation of alveolar cells. The characteristics of the injury are necrotic areas, neutrophilic inflammation, alveolar hemorrage, intra-alveolar and interstitial edema with impairment of alveolar fluid clearance $(31,33)$. This acute inflammatory response is followed by a fibrotic response which starts approximately one week after the application. Yielding $\mathrm{HCl}$ to the airway is characterised with pulmonary artery pressure, pulmonary vascular resistance, shunt fraction and increase in dead space following a rapid increase in airway resistance. While pulmonary capillary wedge pressure and heart rate remain unchanged, cardiac output and mean arterial pressure either remain unchanged or decrease. Main systemic outputs are neutropenia and thrombocytopenia. Lung injury is physiologically characterised with lung compliance and lung volume reduction $(19,28)$.

Sepsis: It is one of the main risk factors of ARDS (30). In order to create sepsis, three major applications are performed (19): 1) injection of live bacteria (administration route, amount, bacterial strain, animal species are important parameters), 2) development of endogenous infection (e.g.: caecal ligation/puncture), and 3) injection of bacterial structures (e.g. endotoxin).

Intravenous bacteria injection: Administration of bacteria can be performed via bolus or permanent infusion (34). Approximately 
one hour after administration, hypotension and the lag-phase of leukopenia is observed; if there is much bacterial retention, death follows septic shock and intravascular coagulation. If animals survive, this continues with a short hemodynamic stabilisation phase within 1-3 hours and then in the lungs, PMN sequestration with microvascular damage, increased pulmonary microvascular permeability, interstitial edema, increased shunt fraction, increased arterial pressure and intravascular thrombosis are observed (19, 35). If bacterial settlement is not high, alveolar epithelium is relatively resistant and low levels of neutrophilic alveolitis and intra-alveolar edema formation are observed. Thus, experimental bacteraemia cannot have all of the histopathological findings including hyaline membrane formation and epithelium damage of ALI/ARDS. On the other hand, the relationship between bacteraemia, sepsis and ARDS is not clear. Although sepsis is a major risk for ARDS, bacteraemia is detected in less than $50 \%$ of clinically diagnosed sepsis patients and when detected, the bacteraemia that is observed is much milder than in most animal models (36).

Lung injury secondary to peritonitis - caecal/ligation/puncture: In the caecal ligation/puncture (CLP) method, the caecum is ligatured and punctured 3-5 times with a needle $(19,37,38)$. Abdominal incision is closed and the animal is allowed to recover. This model results in peritonitis, the systemic markers of which can be detected. The degree of damage depends on the number of punctures made in the caecum and the size of the needle. Generally, animals are progressively leukopenic. Unlike the model in which lipopolysaccharide and live bacteria are injected, following CLP process, cardiac output increases and blood pressure is relatively maintained. After surgery, within 24-30 hours, pulmonary hypertension develops. Blood culture is frequently positive for multiple organisms; growth of enteric gram-negative bacteria (e.g.: Serratia, Enterobacter and Bacteroides) is most frequently observed. Hypoxemia develops in animals and within 18-72 hours, alveolar wall thickening, neutrophil accumulation in the alveolar space and increased permeability in the lungs are observed. Mortality is high (mortality rate for rats is approximately $25 \%$ within 18 hours after operation and $70 \%$ to $90 \%$ within 30 hours after operation).

CLP leads to a lung injury similar to ALI/ARDS, but intraalveolar inflammation and hyaline membrane formation is less dominant.

Most probably, CLP is the only and best organ injury model for sepsis. However, it has some disadvantages like requiring a major surgery (39). Furthermore, the real bacteria inoculum is not known in CLP and it can be affected by the colonic flora among the selected animal models; this situation may cause significant differences in test results.

Endotoxin/lipopolysaccharide (LPS): Lipopolysaccharide is a glycolipid and is located on the outer membrane of gram-negative bacteria. Also, a large part of the biological effects of LPS result from lipid A, which is a part of its content, and LPS has a purified glycolipid structure. In serum, LPS binds to a specific lipopolysaccharide binding protein (LBP) (19). LPS/LBS complex activates monocytes, macrophages and the CD14/TLR-4 receptor complex that is located on other cells and triggers inflammatory mediator production. LPS is an important mediator for gram-negative bacterial sepsis. The administration of systemic lipopolysaccharide is one of the first approaches to form a bacterial sepsis model.
Lipopolysaccharide can be directly and intratracheally administered in an aerosol form, with intranasal deposits or via tracheostomy or endotracheal catheter. Following intravenous lipopolysaccharide administration, the origin of the damage is capillary endothelium; endothelium cell apoptosis occurs rapidly and this is observed before the other tissues are damaged. In this period, while leukopenia, and a decrease in cardiac output and arterial pressure are observed in hemodynamic parameters, pulmonary arterial pressure increases. This lag-phase continues with the development of the number of leukocyte and hemodynamic profile. The changes in the lung become significant within 2-4 hours and hypoxemia is observed. With LPS, histopathological findings of the injury that takes place in the lungs are characterised by diffuse interstitial edema and alveolar exudate formation $(20,40,41)$.

The optimal dose of lipopolysaccharide depends on the bacteria strain and type, and should be determined individually before the study. Animal species, type of LPS, and whether the animals have PIM or not are important factors in lipopolysaccharide-induced lung injury. While low amounts of LPS create pulmonary inflammation in sheep, swine, and cats, it cannot create the same in rodents and dogs. When there is PIM in the animal, even the lowest doses of LPS can create sepsis and lung injury. For animals without PIM, a higher dose of LPS is required. Similarly, there are differences among animal species. For example, while BALB/c mice are very sensitive to LPS, C57BL/6 mice are more resistant. The lung's response to lipopolysaccharide also depends on LPS type. LPS that is created from bacteria with colonies containing O-chain is less pyrogenic, whereas for bacteria without $\mathrm{O}$-chain, it is more pyrogenic. Lipopolysaccharide preparations can be contaminated with structures like bacterial lipoproteins, and this can change the biological effects of LPS (other TLRs are affected by TLR4 which is a LPS receptor). Lipopolysaccharide is quite an appropriate method to study the effects of gram-negative bacteria infractions on humans and animals. It is easy to perform and has high reproducibility rates for test results. It is a potent activator of the immune system and in vitro conditions, toxicity on cells is low. In addition, LPS provides information on the effects of inflammatory responses in bacterial infections. However, this method has some disadvantages, too. The purity of lipopolysaccharide preparations varies, it can be contaminated with bacterial lipoproteins and other bacterial structures. Endothelium and epithelium damage created by lipopolysaccharides is not as severe as in human ARDS. Also, compared to the effects of live bacteria on the lungs, LPS creates an incomplete bacterial damage (it does not contain toxic factors produced by bacteria). In addition to LPS, endotoxin contains a small amount of cell wall proteins, lipids, lipoproteins, and polysaccharides.

Ischemia/reperfusion models: Ischemia/reperfusion (I/R) that takes place in the lungs or remote vascular beds causes lung tissue damage (19). The classical form of lung I/R is a lung injury that develops after a lung transplantation and is a "replacement" response. This response is characterised by non-cardiogenic pulmonary edema, inflammatory infiltrates and hypoxia, and is not related to rejection. Lung injury may also develop after the I/R of extrapulmonary remote areas. For instance, thoracoabdominal aorta aneurysm repair takes an active role in organ I/R; afterwards, ALI may develop. Alveolar epithelium and capillary endothelium are affected in such damage. 
Lung ischemia/reperfusion: lungs are provided with blood by two separate vascular systems (pulmonary and bronchial circulation). Ischemia can be formed by clamping the pulmonary artery; in this case, bronchial circulation is protected, and if the hilum is closed, whole circulation stops. An interesting aspect of this model is that $\mathrm{I} / \mathrm{R}$ in one lung causes inflammatory responses in the other lung. The other lung has slight damage and is characterised by permeability changes and PMN infiltration (19).

There are five main parameters to be considered in pulmonary I/R: 1) Aeration of lung; whether the lung is inflated. If the lung is inflated during the ischemia period, damage decreases; apart from that, atelactasia during the ischemia period causes severe damage (characterised by hemorrhage, edema, and inflammation). Aeration of the lung may also decrease reperfusion injury. 2) Size of the ischemic bed. Failure of one of two systems that provide lung perfusion causes less damage compared to the failure of both. 3) Duration of ischemia/ reperfusion. The duration of both ischemia and reperfusion have an effect on the degree of lung injury. Elongation increases the injury. 4) Experimental preparation. I/R can be performed in vivo or in isolated lung preparations. In vivo models may also enable the study of secondary changes that take place in the other lung. 5) Animal species differences. I/R injury results vary among species. The animals used mostly in pulmonary $\mathrm{I} / \mathrm{R}$ are rabbits, and rats, and dogs. Apart from these animals, sheep, swine, and cats have been used too. Lung I/R is characterised with increased pulmonary vascular permeability and edema, PMN infiltration and sometimes hemorrhage. If the lungs are atelactasic during the ischemic period, and if both pulmonary and bronchial circulation are blocked, lung injury may be more severe.

Extrapulmonary ischaemia/reperfusion: this model is mostly used for rats and mice, but there are also studies that have used rabbits and sheep as well. The model varies depending on the anatomical area (intestine, liver, kidney, hind limb) that underwent ischemia and the duration of ischemia and reperfusion. Lung injury depends on not only the duration of ischemia on these areas and the size of the area that is worked on. Therefore, unless a second intrapulmonary stimulus is added, lung injury is milder. The superior mesenteric artery is the most used vessel for large animals, hind limb ischemia is performed on mice $(40,42,43)$. Generally, the systemic responses observed after I/R are characterised by cardiac output decrease, hypotension, metabolic acidosis and PMN activation. In this model, lung injury depends on the circulated PMNs and proteinaceous alveolar exudates are also observed in lung injury. The advantage of this method is that it imitates the human clinical condition. On the other hand, the I/R of extrapulmonary vascular beds cause a milder lung injury; in order to obtain a more severe injury, a second stimulus is required.

Oleic acid: Oleic acid is the most commonly found free fatty acid in mammals. It is also seen in approximately $50 \%$ of total fatty acids observed in the pulmonary emboli of patients who had long bone trauma. This model is improved to imitate lipid emboli. Lipid emboli is mostly observed as a result of long bone fractures or major traumas (19). Oleic acid directly causes endothelium toxicity. After intravenous administration, endothelium damage starts within a minute. In type I cells, endothelium damage is followed by inflation and necrosis (44). Then, 30 minutes after administration, oleic acid may be detected in the airway. Although the damage mechanism is not known, direct membrane damage appears to be effective.
It is soluble in ethanol or emulsifiable in the blood before application. It can be administered through peripheral or central veins or can be administered directly into the right atrium or pulmonary artery. Since vascular access is required, it is preferred for use in rats or larger animals. With a single dose of oleic acid, maximum ALI is obtained at the $12^{\text {th }}$ hour and towards the $24^{\text {th }}$ hour the effect diminishes. Repeated doses cause fibrosis. It is characterised by necrosis and microvascular thrombosis in the acute phase and followed by a repair phase in which the proliferation of type II cells and fibrotic focus is developed in subpleural spaces. Neutrophilic infiltration is present, but the injury is not dependent on it, endothelium damage plays a critical role. The major advantage of this model is that it can be repeated. The disadvantage is that it requires i.v. administration and experience is needed on animals such as mice. Other disadvantages are the low incidence of lipid injury-induced ARDS and long bone fractures in humans and the fact that the similarity between the injury in this model and sepsis or injuries like aspiration cannot be shown. Since it is a rare ALI/ARDS cause in humans, it is less preferred in mechanism and treatment studies.

Alpha-naphthylthiourea (ANTU): ANTU is a chemical agent developed as rodenticide $(29,40,45)$. It has been determined that it develops ALI depending on the dose and time. Morphological studies performed in electron and light microscopy have shown that the target structure in lung injury mechanisms of ANTU is capillary endothelium cells (46). Endothelium damage causes the elimination of endothelium barrier function and consequently leads to interstitial and alveolar edema development. In a standard experimental model, when the animals (mostly rats and mice) are administered ANTU intraperitoneally, within 4 hours, pulmonary edema and pleural effusion develop reaching a maximum level (47-50). The severity of this damage depends on the dose and time. Edema either disappears within 24-48 hours or develops and causes the death of the rodents (40).

Mechanical Ventilation: Studies have shown that mechanical ventilation may cause pulmonary inflammation and lung injury in animals (ventilator-induced lung injury-VILI) $(19,41)$. The mechanical ventilation model is the only model affected by clinical application performed in humans. Many animal models are created by imitating human ARDS risk factors. However, VILI stems from a treatmentmechanical ventilation. For those people who have clinical risk factors of ARDS, mechanical ventilation is added to the ongoing inflammatory process in the lungs. It is necessary to separate the ventilator-induced lung injury (the only reason of lung injury is mechanical ventilation) from ventilator-related lung injury (ventilation modifies a cause such as sepsis or acid aspiration). VILI causes mechanical stretching and direct tissue damage via specific intracellular pathway activation. Excessive stretching of the alveolar walls cause endothelium and epithelium damage with interstitial edema. On the other hand, hyaline membrane development and increased permeability requires PMN presence; this shows that in mechanical ventilation-mediated injury, in addition to mechanical injury, inflammatory damage is also required. High level tidal volume ventilation results in alveolar hemorrhage, hyaline membrane formation, neutrophilic infiltration, decrease in compliance and gas-exchange abnormalities. The main advantage of this model is that it is clinically relevant and it provides opportunities to make changes in clinical practice. The disadvantage is that it is a complex model. Furthermore, animals such as mice can be ventilated for a short time, whereas patients in clinics need mechanical ventilation for days or even weeks (19). 
Hyperoxia: It is used as a direct damage cause in animal experiments. At the same time, if there is another underlying cause of damage, the damage is increased. Although the molecular basis of oxygen toxicity is still not known, it is believed that oxygen damage occurs directly by the interaction with molecular oxygen-mediated reactive oxygen derivatives (free radicals) or structures like NO. Normal cellular respiration in mitochondria generates a small amount of oxygen superoxide anion. Superoxide forms hydroxyl radicals, which is highly reactive as a result of reacting with hydrogen peroxide $(19,51)$. Normally, the resulting small amount of superoxide and hydrogen peroxide is removed by antioxidant enzymes such as superoxide dismutase, catalase and glutathione peroxidase. In the case of hyperoxia, mitochondria and other organelles produce increasing amounts of superoxide anion and this amount exceeds the capacity of antioxidant enzymes and depletes cellular glutathione. Additionally, superoxide anion reacts with NO and creates peroxynitrite which is a potent oxidant molecule. The accumulation of these molecules leads to oxidation of proteins and peroxidation of nucleic acids with membrane lipids. Cellular injury mechanism appears as the combination of necrosis and apoptosis. Exposure to normobaric oxygen causes an exudative phase in lungs within 3-4 days which is characterised by the death of alveolar type I cells, inflation of endothelium cells and necrosis, interstitial edema, and alveoli being filled with exudative fluid. In the proliferative phase, proliferation of type II pneumocytes and endothelium cells is observed and they cover the alveolar basal membrane. In rats and mice, exposure to $100 \%$ oxygen for $40-50$ hours results in ALI, if the time is prolonged (60-70 hours), death occurs. It has been observed that sensitivity to hyperoxia varies among animal species $(52,53)$.

Surfactant deficiency (depletion of surfactant with saline lavage): Repeated saline lavage decreases surfactant lipid concentration in alveolar surface fluid and changes alveolar surface tension (54). Pulmonary surfactant is a structure with many important functions and consists of a mixture of protein and phospholipids. By decreasing surface tension, surfactant prevents the collapse of alveolar spaces in low pulmonary volume. In addition to stabilising surfactant, surfactant proteins also modulate the immune defence of the lungs. In this model, cold isotonic saline solution $(0.9 \% \mathrm{NaCl})$ was administered to the lungs through instillation and taken back by aspiration. Lavage activity was continued until the desired level of hypoxia was reached. Some researchers add mechanical ventilation to this model (at high volume and end of PEEP-expiration, without positive pressure), which creates a very similar lung injury to the human lung injury observed in ARDS. Pertaining to the connection between surfactant decrease and lung injury, two mechanisms are considered. One of them facilitates alveolar collapse and creates mechanical damage through the repeated expansion and contraction that occurs during mechanical ventilation; the second one is alveolar host-defence mechanism injury. Lung injury is dependent on PMN and is characterised by increased protein permeability, PMN infiltration in the interstitium and airway, increased cytokine production, and hyaline membrane formation. Except in newborns, surfactant deficiency is not a reason for ALI/ARDS but a result. The reason for surfactant abnormalities in ALI/ ARDS is alveolar epithelium damage and exudation of protein-rich edema fluid into alveolar spaces. Saline lavage causes the depletion of surfactant without any major damage in alveolar epithelium. If saline lavage is followed by mechanical ventilation, epithelium injury occurs. The most important disadvantage of this model is that it requires intubation, mechanical ventilation and general anaesthesia, while the advantage is that it provides the opportunity to study the effects of different ventilation strategies on tissue damage (19).

\section{CONCLUSION}

Although none of the available animal models can exactly imitate human pathology, these models are necessary to understand the pathophysiology of ALI/ARDS and improve new treatment strategies. The clarification of the criteria related to defining lung injury in animals will allow the development of animal models in conformity with the specific experimental protocols and enable to perform more detailed analysis of the data gathered from different animal models.

Peer-review: Externally peer-reviewed.

Author Contributions: Both authors have equally contributed to the preparation of this manuscript.

Conflict of Interest: No conflict of interest was declared by the authors.

Financial Disclosure: The authors declared that this study has received no financial support.

\section{REFERENCES}

1. Ashbaugh DG, Bigelow DB, Petty TL, Levine BE. Acute respiratory distress in adults. Crit Care Resusc 2005; 7: 319-23.

2. Bernard GR, Artigas A, Brigham KL, Carlet J, Falke K, Hudson L, et al. The American-European Consensus Conference on ARDS. Definitions, mechanisms, relevant outcomes, and clinical trial coordination. Am J Respir Crit Care Med 1994; 149: 818-24.

3. ARDS Definition Task Force, Ranieri VM, Rubenfeld GD, Thompson BT, Ferguson ND, Caldwell E, et al. Acute respiratory distress syndrome: the Berlin Definition. JAMA 2012; 307: 2526-33.

4. Mustafa Hikmet Özhan, Gülderen Şahin, Erdoğan Kunter, Arzu Balkan. Respiratory distress - from physiology to treatment. Training Manual Series of Turkish Respiratory Society, 2010, İstanbul, 155-181.

5. Ware LB, Matthay MA. The acute respiratory distress syndrome. N Engl J Med 2000; 342: 1334-49.

6. Milberg JA, Davis DR, Steinberg KP, Hudson LD. Improved survival of patients with acute respiratory distress syndrome (ARDS): 1983-1993. JAMA 1995; 273: 306-9.

7. Artigas A, Bernard GR, Carlet J, Dreyfuss D, Gattinoni L, Hudson L, et al. The American-European concensus conference on ARDS, part 2: Ventilatory, pharmacologic, supportive therapy, study design strategies, and issues related to recovery and remodeling. Acute respiratory distress syndrome. Am J Respir Crit Care Med 1998; 157: 1332-47.

8. Fowler AA, Hamman RF, Good JT, Benson KN, Baird M, Eberle DJ, et al. Adult respiratory distress syndrome: risk with common predispositions. Ann Intern Med 1983; 98: 593-7.

9. Villar J, Blanco J, A-ón JM, Santos-Bouza A, Blanch L, Ambros A, et al. The ALIEN study: incidence and outcome of acute respiratory distress syndrome in the era of lung protective ventilation. Intensive Care Med 2011; 37: 1932-41.

10. Tsushima K, S. King L, R. Aggarwal N, De Gorordo A, R. D'Alessio F, Kubo K. Acute lung injury review. Inter Med 2009; 48: 621-30.

11. Jain R, Dalnogare A. Pharmacological therapy for acute respiratory distress syndrome. Mayo Clin Proc 2006; 81: 205-12.

12. Weinacker $A B$, Vaszar LT. Acute respiratory distress syndrome: physiology and new management strategies. Annu Rev Med 2001; 52: 221-37.

13. Matthay MA, Zimmerman GA, Esmon C, Bhattacharya J, Coller B, Doerschuk CM, et al. Future research directions in acute lung injury. Summary of a National Heart, Lung, and Blood Institute Working Group. Am J Respir Crit Care Med 2003; 167: 1027-35.

14. Bellingan GJ. The pulmonary physician in critical care (part 6): the pathogenesis of ALI/ARDS. Thorax 2002; 57: 540-6.

15. Puneet $P$, Moochhala $S$, Bhatia $M$. Chemokines in acute respiratory distress syndrome. Am J Physiol Lung Cell Mol Physiol 2005; 288: L3-L15. 
16. Tasaka S, Hasegawa N, Ishizaka A. Pharmacology of acute lung injury. Pulm Pharmacol Ther 2002; 15: 83-95.

17. Varisco BM. The pharmacology of acute lung injury in sepsis. Adv Pharmacol Sci 2011; 2011: 254619.

18. Dushianthan A, Grocott MPW, Postle AD, Cusack R. Acute respiratory distress syndrome and acute lung injury. Postgrad Med J 2011; 87: 612-22.

19. Mutate-Bello G, Frevert CW, Martin TR. Animal models of acute lung injury. Am J Physiol Lung Cell Mol Physiol 2008; 295: L379.

20. Matute-Bello G, Downey G, B. Moore B, D. Groshong S, Matthay MA, S. Slutsky A, et al. An Official American Thoracic Society Workshop Report: features and measurements of experimental acute lung injury animals. Am J Respir Cell Mol Biol 2011; 44: 725-38.

21. Rehli M. Of mice and men: species variations of Toll-like receptor expression. Trends Immunol 2002; 23: 375-8.

22. Brain JD, Molina RM, DeCamp MM, Warner AE. Pulmonary intravascular macrophages: their contribution to the mononuclear phagocyte system in 13 species. Am J Physiol Lung Cell Mol Physiol 1999; 276: L146-54.

23. Warner AE. Pulmonary intravascular macrophages. Role in acute lung injury. Clin Chest Med 1996; 17: 125-35.

24. Sone Y, Serikov VB, Staub NC Sr. Intravascular macrophage depletion atenuates endotoxin lung injury in anesthetized sheep. J Appl Physiol 1999; 87: 1354-9.

25. Schneemann $M$, Schoedon $G$. Species differences in macrophage NO production are important. Nat Immunol 2002; 3: 102.

26. Zlotnik A, Yoshie O. Chemokines: a new classification system and their role in immunity. Immunity 2000; 12: 121-7.

27. Martin TR, Matute-Bello G. Experimental models and emerging hypotheses for acute lung injury. Crit Care Clin 2011; 27: 735-52.

28. Matute-Bello G, Matthay MA. Animal models of acute lung injury. http://www.thoracic.org/clinical/critical-care/critical-care-research/animal-models-of-acute-lung-injury.php, 2013

29. Atalay F, Yurdakan G, Yilmaz-Sipahi E. The Effect of the endothelin receptor antagonist tezosentan on acute lung injury induced by alpha-naphthylthiourea in rats. Kaohsiung J Med Sci 2012; 28: 72-8.

30. Öztürk Y, Erkal H, Demirhan R, Arıkan Z. Akut respiratuar Distres Sendromu. Türk Göğüs Kalp Damar Cer Derg 2002; 10: 126-30.

31. Reis LK, Uhlig U, Uhlig S. Models and mechanisms of acute lung injury caused by direct insults. Eur J Cell Biol 2012; 91: 590--601.

32. Raghavendran K, Davidson BA, Mullan BA, Hutson AD, Russo TA, Manderscheid $P A$, et al. Acid and particulate-induced aspiration lung injury in mice: importance of MCP-1. Am J Physiol Lung Cell Mol Physiol 2005; 289: L134-43.

33. Yano T, Deterding RR, Simonet WS, Shannon JM, Mason RJ. Keratinocyte growth factor reduces lung damage due to acid instillation in rats. Am J Respir Cell Mol Biol 1996; 15: 433-42.

34. Alper B. İskit. Experimental Models in Sepsis. Turkish Journal of Intensive Care Medicine 2005; 5: 133-6.

35. Welty-Wolf KE, Carraway MS, Ortel TL, Ghio AJ, Idell S, Egan J, et al. Blockade of tissue factor-factor $X$ binding attenuates sepsis-induced respiratory and renal failure. Am J Physiol Lung Cell Mol Physiol 2006; 290: L21-31.

36. Montgomery AB, Stager MA, Carrico CJ, Hudson LD. Causes of mortality in patients with the adult respiratory distress syndrome. Am Rev Respir Dis 1985; 132: 485-89.

37. Berguer R, Alarcon A, Feng S, Gutt C. Laparoscopic cecal ligation and puncture in the rat. Surg Endosc 1997; 11: 1206-8.

38. Otero-Anton E, Gonzalez-Quintela A, Lopez-Soto A, Lopez-Ben S, Llovo J, Perez LF. Cecal ligation and puncture as a model of sepsis in the rat: In- fluence of the puncture size on mortality, bacteremia, endotoxemia and tumor necrosis factor alpha levels. Eur Surg Res 2001; 33: 77-9.

39. Kuklin V, Sovershaev M, Andreasen T, Skogen V, Ytrehus K, Bjertnaes L. Tezosentan reduces the microvascular filtration coefficient in isolated lungs from rats subjected to cecum ligation and puncture. Crit Care 2005; 9: R677-86.

40. Kaynar G, Yurdakan G, Sipahi EY. Effects of the peripheral benzodiazepine receptor ligand Ro5-4864 in four animal models of acute lung injury. J Surg Res 2013; 182: 277-84.

41. Lopez-Aguilar J, Quilez ME, Marti-Sistac O, Garcia-Martin C, Fuster G, Puig $\mathrm{F}$, et al. Early physiological and biological features in three animal models of induced acute lung injury. Intensive Care Med 2010; 36: 347-55.

42. Tekin IO, Sipahi EY, Comert M, Acikgoz S, Yurdakan G. Low-density lipoproteins oxidized after intestinal ischemia/reperfusion in rats. J Surg Res 2009; 157: e47-54.

43. Gabriela C, Oliveira-Filho RM, Trezena AG, da Silva ZL, Domingos HV, de Arruda MJC, et al. Lung microvascular permeability and neutrophil recruitment are differently regulated by nitric oxide in a rat model of intertinal ischemia-reperfusion. Eur J Pharmacol 2004; 494: 241-9.

44. Inoue $H$, Nakagawa $Y$, Ikemura $M$, Usugi E, Nata M. Molecular-biological analysis of acute lung injury (ALI) induced by heat exposure and/or intravenous administration of oleic acid. Leg Med (Tokyo) 2012; 14: 304-8.

45. Sipahi EY, Hodoğlugil U, Ercan, ZS, Türker, RK. Acute effect of endothelin-1 on lung oedema induced by alpha naphtylthiourea (ANTU). Pharmacol Res 1996; 33: 375-8.

46. Sipahi E, Hodoğlugil U, Ustün H, Zengil H, Türker RK, Ercan ZS. "An unexpected interaction between NG-nitro-L-arginine methyl ester and L-arginine in alpha naphtylthiourea-induced pulmonary oedema in rats" Eur $J$ Pharmacol 1997; 321:45-51.

47. Sipahi E, Ustun H, Niyazi Ayoglu F. Acute effects of thiopental, pentobarbital and urethane on lung oedema induced by alpha naphtylthiourea (ANTU). Pharmacol Res 2002; 45: 235-9.

48. Sipahi EY, Ozel Tekin I, Comert M, Barut F, Ustun H, Sipahi TH. Oxidized low-density lipoproteins accumulate in rat lung after experimental lung edema induced by alpha-naphthylthiourea (ANTU). Pharmacol Res 2004; 50: 585-91.

49. Comert M, Sipahi EY, Ustun H, Isikdemir F, Numanoglu G, Barut F, et al. Morphine modulates inducible nitric oxide synthase expression and reduces pulmonary oedema induced by alpha-naphthylthiourea. Eur J Pharmacol 2005; 511: 183-9.

50. Hancı V, Yurdakan G; Yurtlu S, Turan I, Sipahi EY. The protective effect of dexmedetomidine in a rat model of Alpha-naphthylthiourea (ANTU) induced acute lung injury. J Surg Res 2012; 178: 424-30.

51. Kloot TE, Blanch L, Melynne Youngblood A, Weinert C, Adams AB, Marini JJ. Recruitment maneuvers in three experimental models of acute lung injury. Effect on lung volume and gas exchange. Am J Respir Crit Care Med 2000; 161: 1485-94.

52. Barry BE, Crapo JD. Patterns of accumulation of platelets and neutrophils in rat lungs during exposure to $100 \%$ and $85 \%$ oxygen. Am Rev Respir Dis 1985; 132: 548-55.

53. Bellmeyer A, Martino JM, Chandel NS, Scott Budinger GR, Dean DA, Mutlu GM. Leptin resistance protects mice from hyperoxia-induced acute lung injury. Am J Respir Crit Care Med 2007; 175: 587-94.

54. Lachmann B, Robertson B, Vogel J. In vivo lung lavage as an experimental model of the respiratory distress syndrome. Acta Anaesthesiol Scand 1980; 24: 231-6. 